05,12

\title{
Особенности импульсного перемагничивания высококоэрцитивного материала на основе наночастиц $\varepsilon-\mathrm{Fe}_{2} \mathrm{O}_{3}$
}

\author{
() С.И. Попков ${ }^{1}$, А.А. Красиков ${ }^{1}$, С.В. Семенов ${ }^{1}$, А.А. Дубровский ${ }^{1}$, С.С. Якушкин ${ }^{2}$, \\ В.Л. Кириллов ${ }^{2}$, О.Н. Мартьянов ${ }^{2}$, Д.А. Балаев ${ }^{1}$ \\ ${ }^{1}$ Институт фозики им. Л.В. Киренского ФИЦ КНЦ СО РАН, \\ Красноярск, Россия \\ ${ }^{2}$ Институт катализа им. Г.К. Борескова СО РАН, \\ Новосибирск, Россия \\ E-mail: psi@ksc.krasn.ru
}

Поступила в Редакцию 17 октября 2019 г.

В окончательной редакции 17 октября 2019 г.

Принята к публикации 17 октября 2019 г.

\begin{abstract}
Магнитная структура полиморфной модификации оксида железа $\varepsilon-\mathrm{Fe}_{2} \mathrm{O}_{3}$ является коллинеарной ферримагнитной в диапазоне от комнатной температуры до $\sim 150 \mathrm{~K}$. Далее, при уменьшении температуры в $\varepsilon-\mathrm{Fe}_{2} \mathrm{O}_{3}$ происходит магнитный переход, сопровождающийся значительным уменьшением коэрцитивной силы $H_{C}$, и в области низких температур $\varepsilon-\mathrm{Fe}_{2} \mathrm{O}_{3}$ характеризуется сложной несоразмерной магнитной структурой. В данной работе экспериментально исследованы процессы динамического перемагничивания наночастиц $\varepsilon-\mathrm{Fe}_{2} \mathrm{O}_{3}$ среднего размера $8 \mathrm{~nm}$ в температурном диапазоне $80-300 \mathrm{~K}$, охватывающем различные типы магнитной структуры этого оксида железа. Исследовался объемный материал - ксерогель $\mathrm{SiO}_{2}$ с внедренными в поры наночастицами $\varepsilon-\mathrm{Fe}_{2} \mathrm{O}_{3}$. Для измерения петель магнитного гистерезиса в условиях динамического перемагничивания применена методика импульсных магнитных полей напряженностью $H_{\max }$ до $130 \mathrm{kOe}$, использующая метод разрядки батареи конденсаторов через соленоид. Коэрцитивная сила $H_{C}$ при динамическом перемагничивании заметно превосходит $H_{C}$ для квазистатических условий. Это вызвано процессами суперпарамагнитной релаксации магнитных моментов частиц при импульсном перемагничивании. В диапазоне от комнатной температуры до $\sim 150 \mathrm{~K}$ скорость изменения внешнего поля $d H / d t$ является основным параметром, определяющим поведение коэрцитивной силы в условиях динамического перемагничивания. Именно такое поведение ожидается для системы однодоменных ферро- и ферримагнитных частиц. Во внешних условиях (при температуре $80 \mathrm{~K}$ ), когда магнитная структура $\varepsilon$ - $\mathrm{Fe}_{2} \mathrm{O}_{3}$ является несоразмерной, коэрцитивная сила при импульсном перемагничивании уже неоднозначно зависит от параметра $d H / d t$, и в большой степени определяется величиной максимального приложенного поля $H_{\max }$. Такое поведение, нехарактерное для систем ферримагнитных частиц, уже вызвано динамическими процессами спинов внутри частиц $\varepsilon-\mathrm{Fe}_{2} \mathrm{O}_{3}$ при быстром перемагничивании.
\end{abstract}

Ключевые слова: наночастицы $\mathcal{\varepsilon}-\mathrm{Fe}_{2} \mathrm{O}_{3}$, динамическое перемагничивание, коэрцитивная сила.

DOI: 10.21883/FTT.2020.03.49003.609

\section{1. Введение}

Оксид трехвалентного железа $\varepsilon$ - $\mathrm{Fe}_{2} \mathrm{O}_{3}$ является уникальным материалом, с точки зрения магнитных свойств. Существуя только в виде наночастиц размерами до $\sim 20-30 \mathrm{~nm}$, этот полиморф оксида железа демонстрирует коэрцитивную силу $H_{C}$ порядка $20 \mathrm{kOe}$ при комнатной температуре [1-8], что является рекордом для магнитных наночастиц таких размеров [5]. Это обстоятельство, а также связанное с большой коэрцитивной силой свойство - эффективное поглощение электромагнитных волн в миллиметровом диапазоне $[9,10]$, делают материалы на основе наночастиц $\varepsilon$ - $\mathrm{Fe}_{2} \mathrm{O}_{3}$ перспективными для различных практических применений.

Атомы железа в структуре $\varepsilon-\mathrm{Fe}_{2} \mathrm{O}_{3}$ занимают четыpe неэквивалентные позиции. Согласно недавним работам $[11,12]$, магнитный порядок в $\mathcal{E}-\mathrm{Fe}_{2} \mathrm{O}_{3}$ сохраняется до температур $\sim 850 \mathrm{~K}$ (упорядочены две из четырех подрешеток атомов Fe [11]). В окрестности $\sim 500 \mathrm{~K}$ про- исходит упорядочение уже всех четырех подрешеток в коллинеарную ферримагнитную структуру [11], которая существует в температурном диапазоне до $\sim 150 \mathrm{~K}$ (магнитожесткая фаза). Однако в диапазоне $80-150 \mathrm{~K}$ в результате структурных искажений происходит поэтапный магнитный переход $[13,14]$ в некоторую несоразмерную магнитную структуру [14]. При этом в указанном температурном диапазоне происходит резкое уменьшение коэрцитивной силы. Поэтому, несмотря на двадцатилетнюю историю активных исследований $\left(\varepsilon-\mathrm{Fe}_{2} \mathrm{O}_{3}\right.$ впервые надежно был получен в 1998 г. [15]), ряд вопросов, касающихся понимания магнитной структуры этого оксида остается открытым к настоящему времени. Одной из проблем является магнитное состояние $\varepsilon-\mathrm{Fe}_{2} \mathrm{O}_{3}$ в области низких температур (до $80 \mathrm{~K}$ ).

Импульсное перемагничивание (основной метод, применяемый в данной работе), является частным случаем исследования динамического магнитного гистерезиса 
(ДМГ). Последнее подразумевает изучение влияния частоты $v$ переменного магнитного поля

$$
H=H_{\max } \sin (2 \pi v t)
$$

на параметры петли гистерезиса $\left(H_{C}\right.$, площадь петли, и т.п.). Если для объемных материалов динамический гистерезис, в первую очередь, определяется процессами в доменной структуре, то для однодоменных ферро- и ферримагнитных частиц динамический гистерезис связан с релаксационными процессами магнитного момента частицы, и зависит от размеров частиц, их магнитной структуры и магнитной анизотропии [16-18]. Поведение ДМГ связано с приложениями в области гипертермии $[19,20]$ и магнитной памяти [21,22]. Поэтому исследование ДМГ является одной из важных и перспективных методик изучения магнитного состояния в системах наночастиц.

Отметим один факт, существенный при исследовании ДМГ. В обычных установках, генерирующих переменное магнитное поле, есть существенные ограничения на значения максимального приложенного поля $H_{\max }$ и частоты $v$. Эти ограничения связаны с мощностью, выделяемой в соленоиде. Поэтому для частот порядка $10^{2}-10^{3} \mathrm{~Hz}$ трудно достичь величин поля $H_{\max }$ более $\sim 10^{3}$ Ое. Это обстоятельство затрудняет исследование ДМГ в материалах, характеризующихся большими значениями магнитной анизотропии, поскольку, если $H_{\max }$ существенно меньше поля необратимого поведения намагниченности $H_{\text {irr }}$, то результирующая петля гистерезиса будет частной. Следовательно, и полученные параметры $\left(H_{C}\right.$, остаточная намагниченность) будут, в основном, определяться эффектами частной петли гистерезиса. Альтернативой стандартным методам исследования ДМГ является использование импульсных полей, при которых выделение мощности ограничивается одиночным циклом изменения внешнего поля, и появляется возможность увеличивать максимальное приложенное поле до величин порядка сотен $\mathrm{kOe}$.

Для $\varepsilon-\mathrm{Fe}_{2} \mathrm{O}_{3}$ величина $H_{\text {irr }}$ достигает $\sim 60 \mathrm{kOe}[3,5,23]$, поэтому исследования динамического перемагничивания наночастиц $\varepsilon-\mathrm{Fe}_{2} \mathrm{O}_{3}$ возможно проводить только с использованием импульсных полей. Ранее нами были исследованы процессы динамического перемагничивания наночастиц $\varepsilon-\mathrm{Fe}_{2} \mathrm{O}_{3}$ среднего размера $\sim 10 \mathrm{~nm}$ при температурах 300 и $77 \mathrm{~K}$ [24]. Зависимости коэрцитивной силы как функции скорости изменения внешнего поля $d H / d t$ были проанализированы на основании теоретической модели для динамического перемагничивания ферромагнитных наночастиц $[25,26]$. Это позволило однозначно выделить вклад поверхностной магнитной анизотропии в формирование динамических свойств наночастиц данного оксида [24]. Модель [25,26] хорошо описала результаты эксперимента при комнатной температуре, в то время как при $77 \mathrm{~K}$ наблюдалось значительное расхождение между теоретической и экспериментальной зависимостями $H_{C}$ от $d H / d t$ [24]. С целью установления картины температурной эволюции динамической коэрцитивной силы, а также выявления причин указанного расхождения модели и эксперимента при $T=77 \mathrm{~K}$ в данной работе проведены исследования импульсного перемагничивания материала на основе наночастиц $\varepsilon-\mathrm{Fe}_{2} \mathrm{O}_{3}$ (средний размер $\sim 8 \mathrm{~nm}$ ). Диапазон, в котором проведены измерения, составляет $80-300 \mathrm{~K}$, что охватывает как магнитожесткую фазу (температурный диапазон ее существования $150-500 \mathrm{~K}$ ), так и сложную (в точности неустановленную к настоящему времени) несоразмерную магнитную структуру при $80 \mathrm{~K}$, формирующуюся после магнитного перехода, происходящего в температурном интервале $80-150 \mathrm{~K}$.

\section{2. Эксперимент}

\section{1. Приготовление и характеризация образца наночастиц $\varepsilon-\mathrm{Fe}_{2} \mathrm{O}_{3} /$ ксерогель $\mathrm{SiO}_{2}$}

Исследовался образец, содержащий $20 \mathrm{wt} . \% \varepsilon-\mathrm{Fe}_{2} \mathrm{O}_{3}$ в матрице ксерогеля $\mathrm{SiO}_{2}$. Образец был получен методом, впервые реализованным и подробно описанным в работе [27]. Порошковая дифрактограмма была получена на дифрактометре D8 Advance Bruker (Германия) в излучении $\mathrm{Cu} K_{\alpha}$ при длине волны $1.5418 \AA ̊$. Микрофотографии частиц были получены с использованием электронного микроскопа JEOL JEM-2010 при ускоряющем напряжении $200 \mathrm{kV}$ с разрешением $1.4 \AA$.

Согласно данным рентгеноструктурного анализа, все наблюдаемые дифракционные пики принадлежат структуре $\varepsilon-\mathrm{Fe}_{2} \mathrm{O}_{3}-$ см. рис. $1, a$. По данным просвечивающей электронной микроскопии высокого разрешения (рис. 1,c) было получено, что средний размер частиц составляет $8 \mathrm{~nm}$. Гистограмма распределения частиц по размерам, полученная из большого количества снимков, приведена на рис. 1, $b$. Кроме того, анализ мессбауэровских спектров показал, что других полиморфных модификаций оксида железа, кроме $\varepsilon-\mathrm{Fe}_{2} \mathrm{O}_{3}$, в образце не содержится [27,28].

Отметим, что образцы, в которых наночастицы $\varepsilon-\mathrm{Fe}_{2} \mathrm{O}_{3}$ содержатся в матрице ксерогеля $\mathrm{SiO}_{2}$, представляют собой объемные пластинки сантиметрового размера. Это дает возможность проводить измерения в магнитных полях без специальных мер по фиксации наночастиц, что является необходимым в случае порошкообразных образцов.

\section{2. Измерение квазистатических магнитных свойств}

Квазистатические магнитные свойства измерялись на вибрационном магнетометре [29] (VSM). Температурные зависимости намагниченности $M(T)$ измерены в условиях охлаждения в нулевом внешнем поле (zero field cooled - ZFC) и охлаждения в поле (field cooled - FC). Полевые зависимости намагниченности $M(H)$ измерены в условиях ZFC. Скорость изменения поля $(d H / d t)_{\mathrm{VSM}}$ 

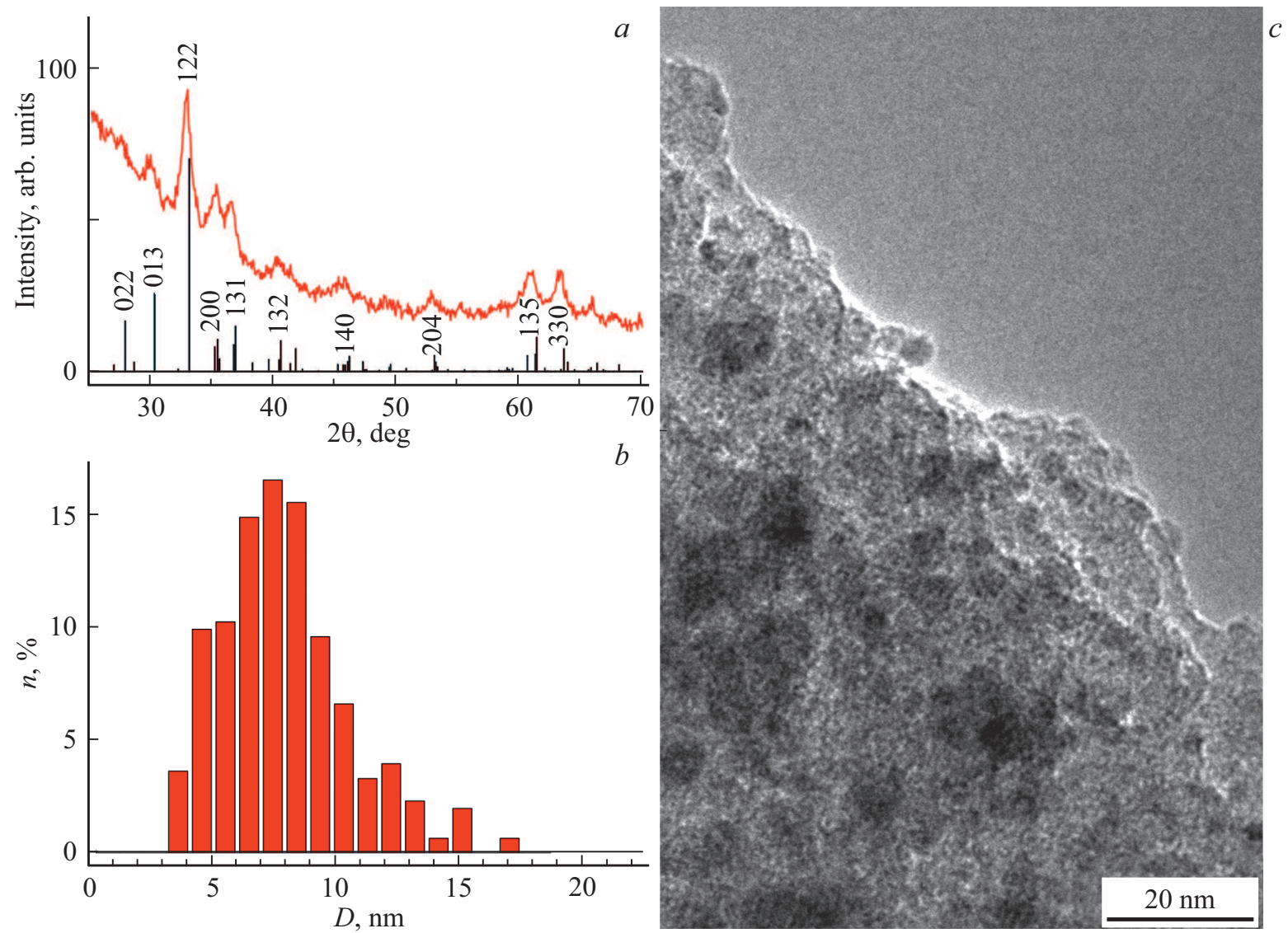

Рис. 1. Дифрактограмма исследованного образца $(a)$, распределение частиц по размерам $(b)$ и типичная микрофотография частиц $(c)$, полученная просвечивающей электронной микроскопией.

при измерении квазистатических петель гистерезиса $M(H)$ составляла $\approx 50 \mathrm{Oe} / \mathrm{s}$. Для получения зависимости $H_{C}$ от максимального приложенного поля $H_{\max }$ измерялось семейство частных петель гистерезиса с последовательно увеличивающейся величиной $H_{\max }$. Значения намагниченности нормированы на массу оксида железа в образце.

\section{3. Измерение петель ДМГ при импульсном перемагничивании}

Для исследований ДМГ использовалась оригинальная установка сильных импульсных полей Института физики им. Л.В. Киренского СО РАН, использующая метод разрядки батареи конденсаторов через соленоид. Обычно колебательный процесс в подобных установках останавливается тиристором после первой полуволны, в то время как для измерения петель гистерезиса намагниченности требуется не менее двух полуволн. Для формирования второй полуволны, в схему установки встречнопараллельно тиристору были включены диоды. На рис. 2 представлены типичные зависимости поля в соленоиде от времени при различных значениях максимального поля (определяемого напряжением зарядки конденсаторов) и длительности импульса, времени полуволны -
$\tau_{P}$. Величина $\tau_{P}$ изменялась путем коммутации блоков батареи конденсаторов на различные емкости. Измерения проводились при значениях $\tau_{P}$, равных 4, 8 и $16 \mathrm{~ms}$.

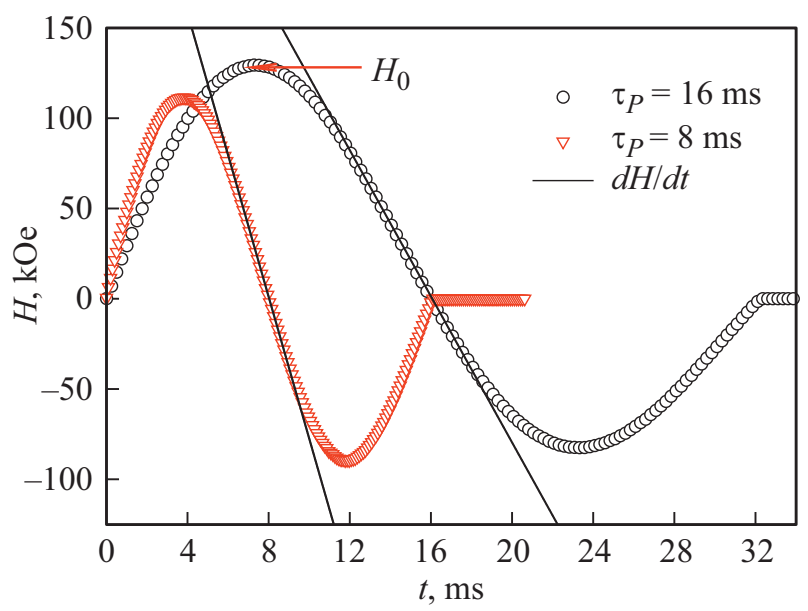

Рис. 2. Примеры типичных зависимостей поля соленоида от времени в импульсной методике при различных значениях максимального приложенного поля $H_{0}$ и указанных значениях длительности импульса $\tau_{P}$. Наклоны прямых линий соответствуют скорости изменения поля $d H / d t$ в момент перемагничивания образца. 
Нулевое значение поля в момент $t=\tau_{P}$ обусловлено закрытием блоков тиристоров.

Для измерения намагниченности использовался индукционный датчик, представляющий собой систему коаксиальных скомпенсированных катушек, в которую помещался образец. Индуцируемый в катушках сигнал усиливался и записывался цифровым запоминающим осциллографом. Измерения проводились в диапазоне температур 80-300 К. При постоянной температуре в каждом последующем измерении величина $H_{\max }$ была больше предыдущей. Данные, полученные в импульсных полях, сопоставлялись по величине намагниченности с петлями гистерезиса, измеренными методикой VSM.

\section{3. Результаты и обсуждение}

На рис. 3 приведены температурные зависимости намагниченности $M(T)$ исследованного образца в режимах ZFC и FC. Наблюдаемое немонотонное поведение зависимостей $M(T)$ (максимумы в окрестности $110-120 \mathrm{~K})$ не является проявлением процессов суперпарамагнитной блокировки. Для частиц $\varepsilon-\mathrm{Fe}_{2} \mathrm{O}_{3}$ размерами $6 \mathrm{~nm}$ и более температура суперпарамагнитной блокировки значительно превышает комнатную температуру [23,28,30,31]. Отчетливо видимые на рис. 3 аномалии в окрестности $120 \mathrm{~K}$ и изменение характера зависимостей $M(T)$ окрестности $80 \mathrm{~K}$ отражают известный для $\varepsilon-\mathrm{Fe}_{2} \mathrm{O}_{3}$ магнитный переход, происходящий в температурном интервале 80-150 K $[3,13,14]$.

Этот магнитный переход проявляется и в температурном поведении коэрцитивной силы. На рис. 4 приведена зависимость $H_{C}(T)$, полученная из измерений петель магнитного гистерезиса в квазистатических условиях (VSM). Достаточно резкое уменьшение коэрцитивной силы, связанное с магнитным переходом, начинается от $\sim 150 \mathrm{~K}$ (при понижении температуры). Отметим,

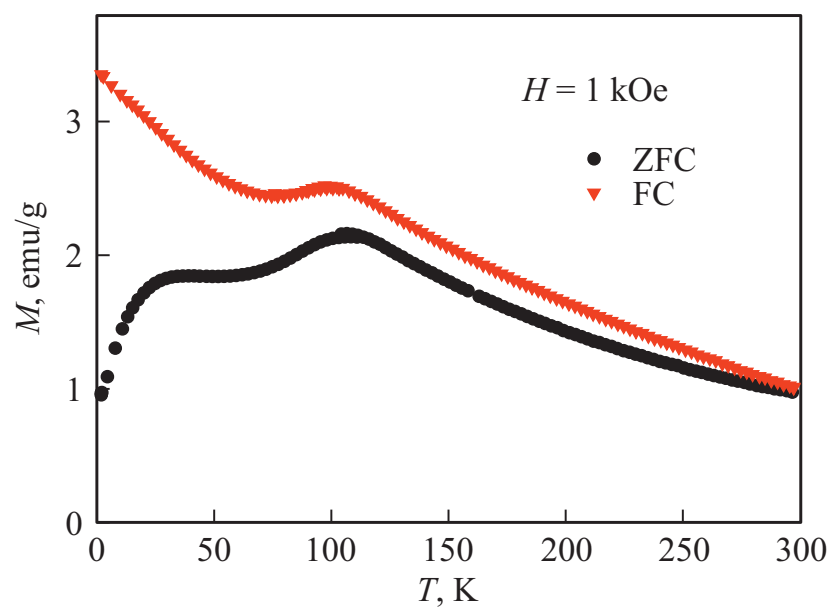

Pис. 3. Температурные зависимости намагниченности $M(T)$ исследованного образца наночастиц $\varepsilon-\mathrm{Fe}_{2} \mathrm{O}_{3}$ в различной и термомагнитной предыстории в поле $H=1 \mathrm{kOe}(\mathrm{ZFC}-$ охлаждение в нулевом внешнем поле, $\mathrm{FC}$ - охлаждение в поле).

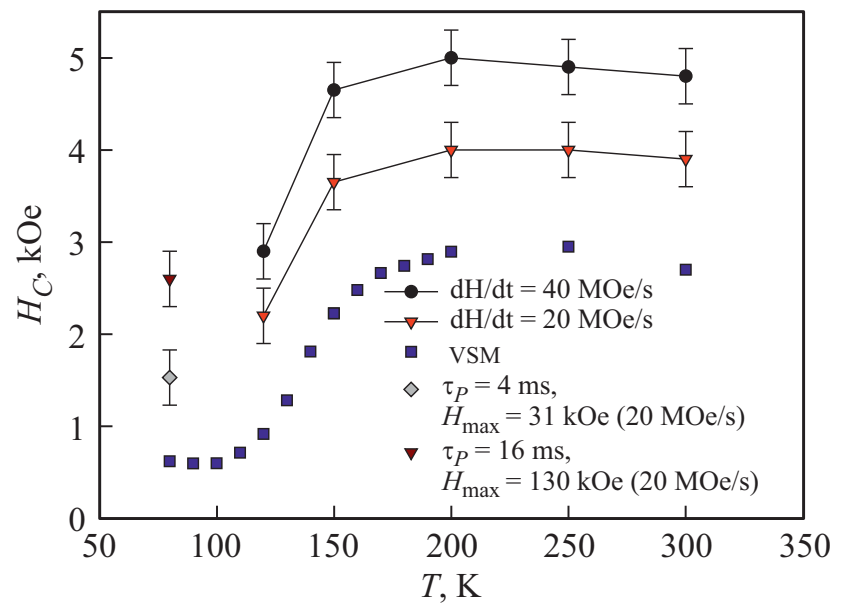

Рис. 4. Температурная эволюция коэрцитивной силы $H_{C}$ для квазистатических измерений гистерезиса намагниченности (VSM) и при импульсном перемагничивании при приведенных на рисунке параметрах.

что относительно небольшие значения $H_{C}(\sim 3 \mathrm{kOe}$ при комнатной температуре) для исследуемого образца определяются достаточно малым размером частиц $(8 \mathrm{~nm}) \quad[5,23,32,33]$. Частицы $\varepsilon-\mathrm{Fe}_{2} \mathrm{O}_{3}$ размерами $\sim 10$ и $20 \mathrm{~nm}$ демонстрируют коэрцитивную силу порядка 10 и $20 \mathrm{kOe} \mathrm{соответственно} \mathrm{[5,33].}$

При измерениях в импульсном поле (см. рис. 2) регистрируются части петли гистерезиса в диапазонах полей от (i) $H=0$ до $H_{\max }$, затем (ii) от $H_{\max }$ до отрицательного значения поля $-H_{m}$, несколько меньшего $\left|H_{\max }\right|$ (затухающие колебания в LCR-контуре), и затем, (iii) от $-H_{m}$ до нулевого значения поля. Наиболее информативной частью зависимости $M(H)$ этой методики является область (ii), и далее, при анализе данных по импульсному перемагничиванию, мы будем оперировать с этой частью зависимости $M(H)$ (в области отрицательных полей). На рис. 5, $a$ и 6, $a$ приведены типичные гистерезисные зависимости $M(H)$ исследованных образцов, полученные при $T=80 \mathrm{~K}$ и $300 \mathrm{~K}$ соответственно. На этих рисунках совместно приведены данные квазистатической магнитометрии VSM (при $H_{\max }=60 \mathrm{kOe}$ ) и зависимости $M(H)$, полученные методикой импульсного магнетометра при указанных на рисунках значениях $\tau_{P}$ и $H_{\max }$. Рис. $5, b$ и $6, b$ иллюстрируют типичное поведение петель гистерезиса (рис. $5, b-T=80 \mathrm{~K}$, рис. $6, b-$ $T=300 \mathrm{~K}$ ) для использованных методик в окрестности начала координат. Петли гистерезиса, полученные при импульсном перемагничивании, заметно шире таковых для методики VSM. Для всех полученных данных при импульсном перемагничивании наблюдается уширение петель гистерезиса как при увеличении $H_{\max }$, так и при уменьшении времени импульса $\tau_{P}$. Далее будем оперировать с величиной коэрцитивной силы $H_{C}$, в области отрицательных значений поля, полученной, как точка пересечения зависимости $M(H)$ с осью абсцисс в области отрицательных полей. 

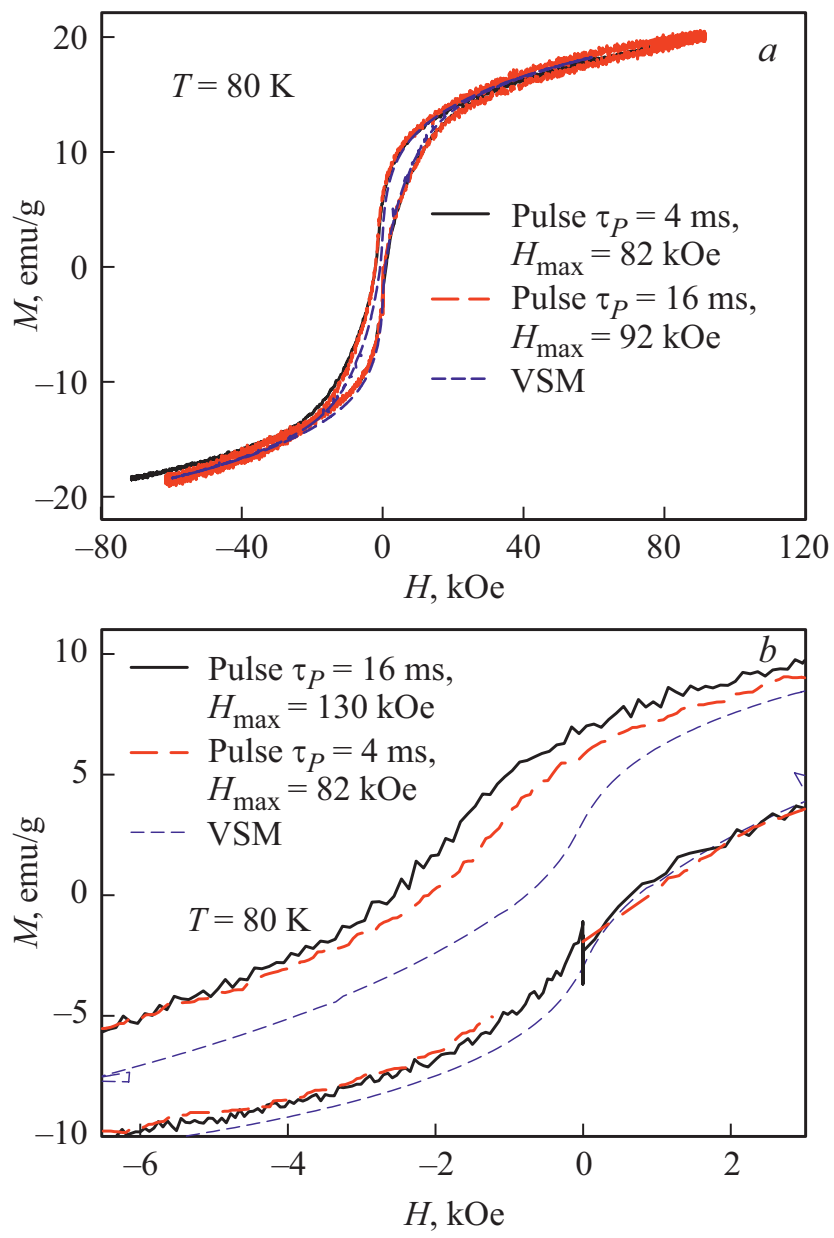

Рис. 5. Типичные петли гистерезиса (a) исследованного образца наночастиц $\varepsilon$ - $\mathrm{Fe}_{2} \mathrm{O}_{3}$, полученные методикой VSM и в импульсных полях при различных параметрах импульса при температуре $300 \mathrm{~K} .(b)$ - поведение зависимостей $M(H)$ в окрестности начала координат. Значения $\tau_{P}$ и $H_{\max }$, приведены на рисунках.

При теоретическом рассмотрении ДМГ площадь петли гистерезиса определяется (при прочих равных условиях) частотой переменного поля $[16,17,25,26]$ и значением $H_{\max }$. Оба этих параметра определяют скорость изменения внешнего поля $d H(t) / d t$, что при выполнении гармонического закона (1), приводит к следующему выражению: $d H(t) / d t=2 \pi v H_{\max } \cos (2 \pi v t)$. Если рассматривать только один параметр петли гистерезиса — коэрцитивную силу, то при условии $H_{C} \ll H_{\max }$ величина $d H / d t$ с хорошей точностью соответствует наклону касательной к зависимости $H(t)$, как показано на рис. 2. В наших экспериментах варьируются значения $H_{\max }$ и $\tau_{P}$; в обозначениях, принятых для импульсных методик, $v=1 / 2 \tau_{P}$ (см. также рис. 2). Следовательно, в окрестности $H=0$ скорость изменения внешнего поля будет определяться как:

$$
d H / d t=H_{\max } / 2 \tau_{P}
$$

Сфокусируем внимание на зависимости коэрцитивной силы от $d H / d t$, а также от варьируемых параметров импульса $-\tau_{P}$ и $H_{\max }$. Рис. 7 иллюстрирует зависимости $H_{C}$ от $d H / d t$ и (рис. 7,a,c) $H_{C}$ от $H_{\max }$ (рис. 7, $\left.b, d\right)$ для зависимостей $M(H)$ полученных при температурах 80 и $300 \mathrm{~K}$. Для температуры $300 \mathrm{~K}$ в координатах $H_{C}$, $d H / d t$ данные для двух значений продолжительности импульса укладываются на одну функциональную зависимость - рис. 7,a. Это и дает основание рассматривать наночастицы $\varepsilon$ - $\mathrm{Fe}_{2} \mathrm{O}_{3}$ как однодоменные ферримагнитные частицы [3,24], и применять к полученным результатам теоретическую модель [25,26], развитую для ферромагнитных частиц. И, как следствие выполнения выражения (2), экспериментальные данные в координатах $H_{C}, H_{\max }$ разбиваются на две зависимости, соответствующие различным продолжительностям импульca: при $H_{\max } \approx$ const $H_{C}\left(\tau_{P}=8 \mathrm{~ms}\right)>H_{C}\left(\tau_{P}=16 \mathrm{~ms}\right)$, см. рис. 7, $b$. Описываемое поведение (однозначность зависимости $\left.H_{C}(d H / d t)\right)$ имеет место для данных
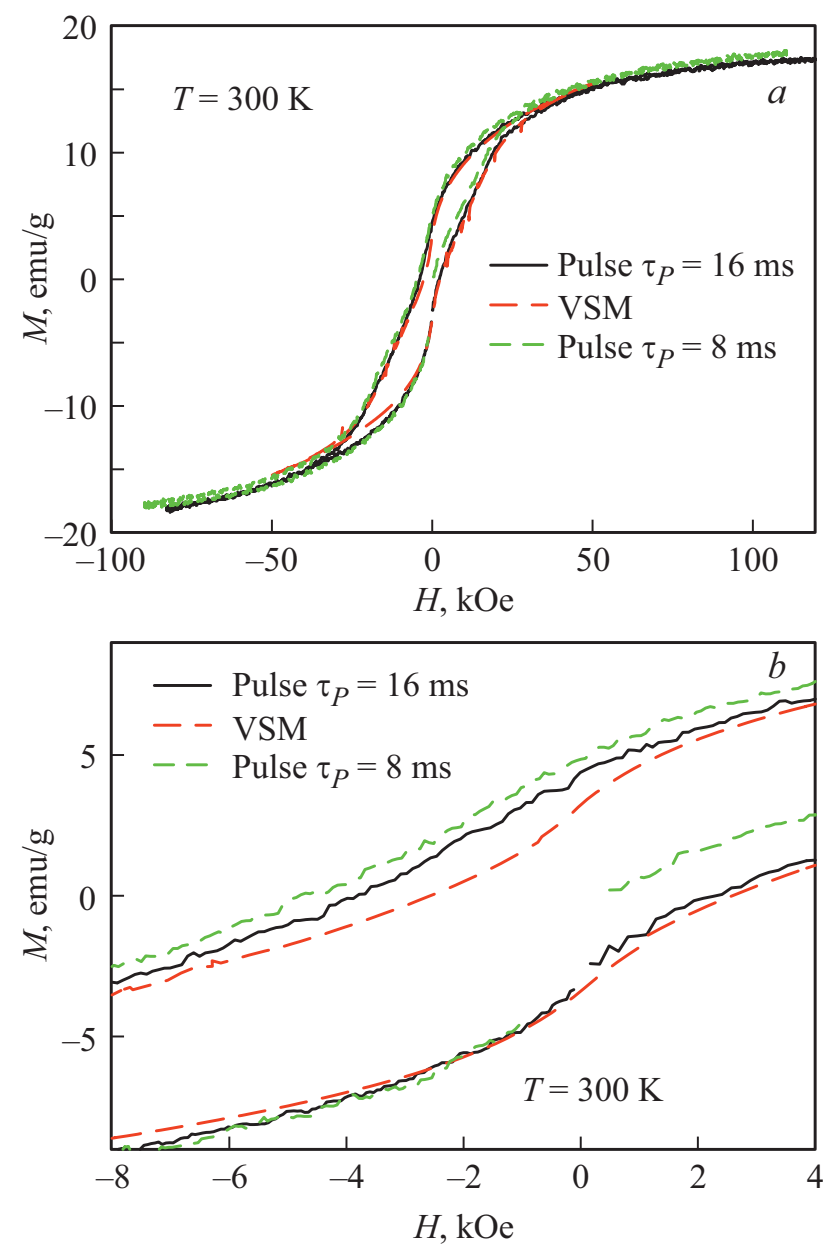

Рис. 6. Типичные петли гистерезиса (a) исследованного образца наночастиц $\varepsilon-\mathrm{Fe}_{2} \mathrm{O}_{3}$, полученные методикой VSM и в импульсных полях при различных параметрах импульса при температуре $80 \mathrm{~K} .(b)$ - поведение зависимостей $M(H)$ в окрестности начала координат. Значения $\tau_{P}$ и $H_{\max }$, приведены на рисунках. 

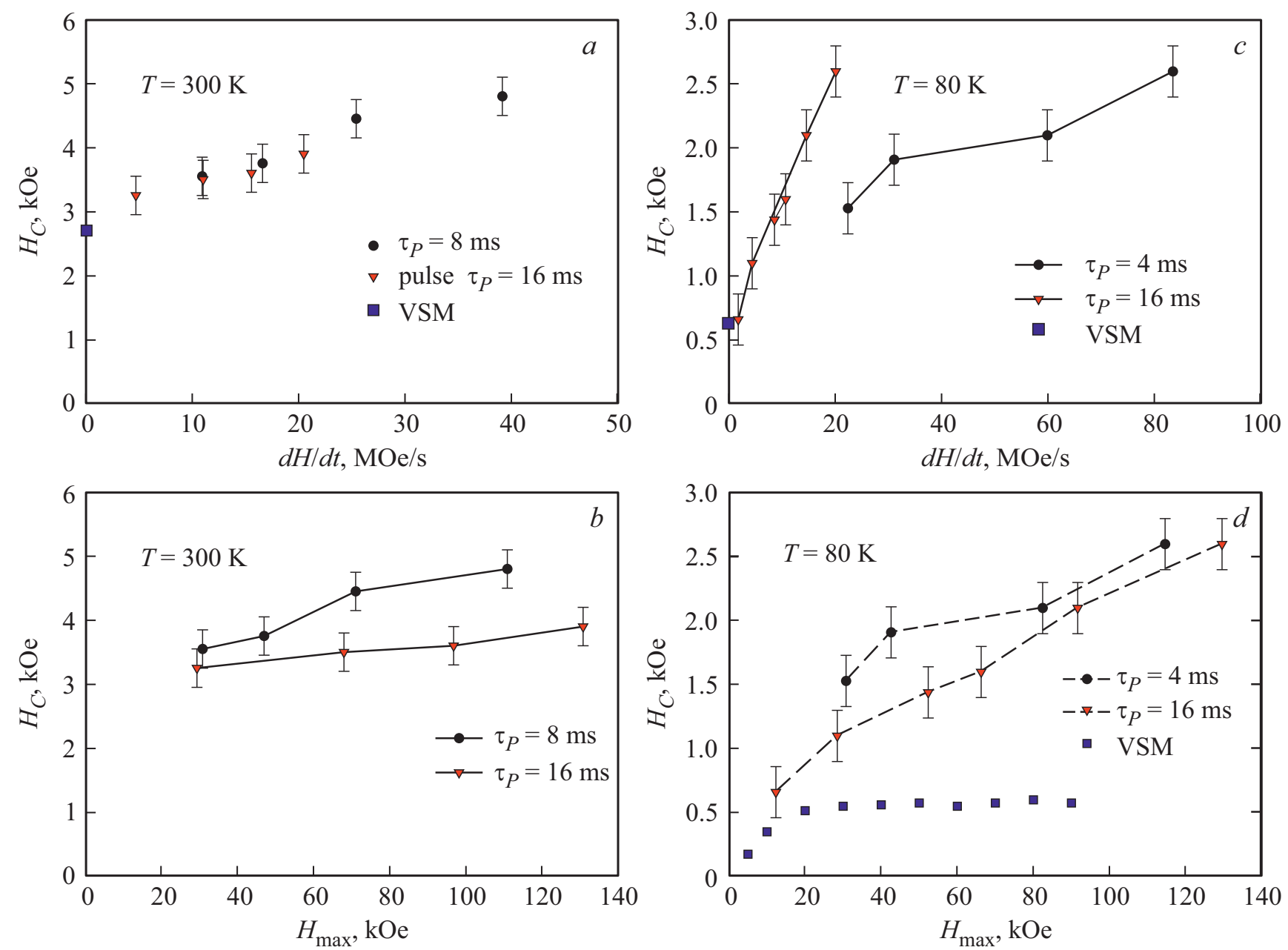

Рис. 7. Поведение коэрцитивной силы $H_{C}$ при импульсном перемагничивании от параметров $d H / d t(a, c)$ и $H_{\max }(b, d)$ для образца наночастиц $\varepsilon-\mathrm{Fe}_{2} \mathrm{O}_{3}$ при температурах $300(a, b)$ и $80 \mathrm{~K}(c, d)$ при указанных параметрах значениях времени импульса $\tau_{P}$. На $(b, c, d)$ данные по одинаковой длительности импульса сгруппированы соединяющими линиями. На $(a, c, d)$ приведены также данные, полученные методикой VSM.

в температурной области от комнатной температуры до $120-150 \mathrm{~K}$. Следовательно, именно скорость перемагничивания $d H / d t$ для ферримагнитных наночастиц является параметром, однозначно определяющим коэрцитивную силу однодоменных ферро- и ферримагнитных наночастиц в процессах динамического перемагничивания.

Для данных, полученных в области ниже магнитного перехода - при $80 \mathrm{~K}$, наблюдается принципиально другое поведение коэрцитивной силы от параметров импульса. Зависимость $H_{C}$ от $d H / d t$ уже на является однозначной функцией для данных при различных значениях длительности импульса - см. рис. 7, $c$. Напротив, данные на рис. 7,c разбиваются на две достаточно далеко друг от друга расположенные зависимости для каждого значения $\tau_{P}$. В то же время, в координатах $H_{C}$, $H_{\text {max }}$ группы точек для различных величин $\tau_{P}$ расположены близко друг другу (рис. 7,d), хотя неравенство $H_{C}\left(\tau_{P}=4 \mathrm{~ms}\right)>H_{C}\left(\tau_{P}=16 \mathrm{~ms}\right)$ имеет место. Можно заключить, что для данных, полученных при темпера- туре $80 \mathrm{~K}$ универсального параметра, определяющего коэрцитивную силу, нет.

Поведение, схожее с данными, полученными для температуры $80 \mathrm{~K}$ (рис. 7, $c, d$ ), наблюдалось нами для антиферромагнитных наночастиц $\mathrm{NiO}$ [34] и ферригидрита [35]. В случае наноразмерных частиц антиферромагнетика гистерезис намагниченности обусловлен существованием у таких объектов нескомпенсированного магнитного момента благодаря дефектам структуры и поверхностным эффектам [36-40]. Наличие не одной (антиферромагнитной), а двух, или более магнитных подсистем в антиферромагнитно упорядоченных наночастицах, а также взаимодействие указанных подсистем, могут быть причиной наблюдаемых высоких полей необратимого поведения кривых намагничивания $H_{\text {irr }}$ таких объектов [41-43]. Это приводит к тому, что зависимость $H_{C}\left(H_{\max }\right)$ для наночастиц антиферромагнетика не выходит на насыщение, и петли магнитного гистерезиса остаются открытыми при $H_{\max }<H_{\text {irr }}$. И, как следствие, в импульсных полях значение максимального 
приложенного поля $H_{\max }$ играет бо́льшую роль, чем в выражении (2).

Указанная аналогия с антиферромагнитными наночастицами была бы разумным объяснением наблюдаемого поведения для $\varepsilon-\mathrm{Fe}_{2} \mathrm{O}_{3}$, если признать, что именно дефекты в данном оксиде железа приводят к наличию у частиц магнитного момента. Однако гистерезис намагниченности для частиц $\varepsilon-\mathrm{Fe}_{2} \mathrm{O}_{3}$ размерами более $6 \mathrm{~nm}$ наблюдается во всем диапазоне температур (включая и обсуждаемую температуру $80 \mathrm{~K}$ ). Более того, для квазистационарных условий при $80 \mathrm{~K}$ зависимость $H_{C}\left(H_{\max }\right)$ выходит на насыщение в полях, порядка 30-40 kOе (данные VSM на рис. 7,d), что не позволяет проводить однозначную аналогию между антиферромагнитными наночастицами и наночастицами $\mathcal{\varepsilon}-\mathrm{Fe}_{2} \mathrm{O}_{3}$. При температурах 80 и $300 \mathrm{~K}$, для которых обсуждаются полученные зависимости $H_{C}(d H / d t)$ и $H_{C}\left(H_{\max }\right)$, магнитная структура $\varepsilon-\mathrm{Fe}_{2} \mathrm{O}_{3}$ различна. Если при комнатной температуре $\varepsilon-\mathrm{Fe}_{2} \mathrm{O}_{3}$ является коллинеарным ферримагнетиком, и поведение гистерезиса намагничивания может быть описано в рамках классического рассмотрения модели Стонера-Вольфарта [44], то после магнитного перехода в диапазоне $80-150 \mathrm{~K}$ магнитная структура $\varepsilon-\mathrm{Fe}_{2} \mathrm{O}_{3}$ становится несоразмерной $[3,14]$. Поэтому можно заключить, что смена магнитной структуры кардинально сказывается на поведении коэрцитивной силы при импульсном перемагничивании.

Вышесказанное объясняет причины несоответствия экспериментальной зависимости $H_{C}(d H / d t)$, полученной в работе [24] для частиц $\varepsilon-\mathrm{Fe}_{2} \mathrm{O}_{3}$ (размер $\sim 10 \mathrm{~nm}$ ) при температуре $77 \mathrm{~K}$ и теоретической моделью $[25,26]$, рассматривающей однодоменные ферро- или ферримагнитные частицы. Для систем однодоменных ферроили ферримагнитных частиц результирующая (гистерезисная) кривая намагничивания связана с процессами выстраивания магнитных моментов всех частиц по полю (конкуренция зеемановской энергии и энергии магнитной анизотропии). При этом величина магнитного момента отдельной частицы считается неизменной (определяется магнитным упорядочением внутри частицы). Для сложной несоразмерной магнитной структуры вполне возможен и дополнительный вклад частичный поворот спинов атомов железа уже внутри частицы. И этот процесс может быть динамическим, т.е., угол поворота спинов (с учетом усреднения при хаотической ориентации кристаллографических осей) в несоразмерной магнитной структуре будет существенно зависеть от времени перемагничивания. Тогда логично, что параметр $d H / d t$ не будет основным параметром, определяющим процессы намагничивания и перемагничивания, а время импульса играет бо́льшую роль, чем для случая ферримагнитного порядка внутри частицы. Отметим, что в работах $[13,45]$ наблюдались аномальные зависимости АC восприимчивости $\chi$ от частоты приложенного поля в области температур 80-100 K: максимум зависимостей $\chi(T)$ смещался в область низких температур с увеличением частоты переменного поля.
Это косвенно подтверждает наши аргументы о том, что именно изменение магнитной структуры приводит к наблюдаемому нетипичному для однодоменных ферримагнитных частиц поведению коэрцитивной силы в условиях динамического перемагничивания.

Температурная эволюция коэрцитивной силы для исследованного образца в условиях импульсного перемагничивания показана на рис. 4. На этом рисунке совместно с зависимостью $H_{C}(T)$, полученной методикой VSM, приведены данные при значениях $d H / d t$, равных 20 и $40 \mathrm{MOe} / \mathrm{s}$. Видно, что в диапазоне от комнатной температуры до $\sim 120-150 \mathrm{~K}$ поведение динамической коэрцитивной силы схоже с таковым для квазистатических условий. Достаточно резкое уменьшение коэрцитивной силы начинается от $\sim 150 \mathrm{~K}$ (при понижении температуры), и это связано с магнитным переходом. Поскольку для температуры $80 \mathrm{~K}$ параметр $d H / d t$ уже не является универсальным для описания динамической коэрцитивной силы, для данной температуры на рис. 4 приведены значения $H_{C}$, полученные при $H_{\max }=31$ и $130 \mathrm{kOe}$ и $\tau_{P}=4$ и $\tau_{P}=16 \mathrm{~ms}$ соответственно (эти параметры соответствуют величине $d H / d t$, равной $20 \mathrm{MOe} / \mathrm{s}$ ).

\section{4. Заключительные замечания}

Таким образом, в данной работе экспериментально исследованы процессы перемагничивания наночастиц $\varepsilon-\mathrm{Fe}_{2} \mathrm{O}_{3}$ средним размером $8 \mathrm{~nm}$ в импульсных полях в диапазоне температур 80-300 K. В области от комнатной температуры до $150 \mathrm{~K}$, в которой $\varepsilon$ - $\mathrm{Fe}_{2} \mathrm{O}_{3}$ обладает коллинеарной ферримагнитной структурой, имеет место поведение, типичное для однодоменных ферро- и ферримагнитных частиц - коэрцитивная сила увеличивается с ростом скорости изменения поля $d H / d t$, и именно этот параметр является основным при описании процессов динамического перемагничивания. Температурная эволюция динамической коэрцитивной силы подобна таковой для условий квазистатического перемагничивания. При температуре $80 \mathrm{~K}$, что ниже известного магнитного перехода в $\varepsilon$ - $\mathrm{Fe}_{2} \mathrm{O}_{3}$ (происходящего в диапазоне $80-150 \mathrm{~K}$ ), поведение коэрцитивной силы уже не может быть описано одним параметром $d H / d t$. Большую, если не решающую роль, здесь играет максимальное приложенное поле $H_{\max }$. Можно констатировать, что кардинальное изменение магнитной структуры, вызванное магнитным переходом, уже не позволяет рассматривать частицы $\varepsilon-\mathrm{Fe}_{2} \mathrm{O}_{3}$ как однодоменные ферримагнитные. Обнаруженное поведение является отличительной чертой изменения магнитной структуры $\mathcal{\varepsilon}-\mathrm{Fe}_{2} \mathrm{O}_{3}$ после магнитного перехода, что важно для понимания магнитной фазовой диаграммы $\varepsilon-\mathrm{Fe}_{2} \mathrm{O}_{3}$.

\section{Финансирование работы}

Исследование выполнено при финансовой поддержке Российского фонда фундаментальных исследований, 
Правительства Красноярского края, Красноярского краевого фонда науки в рамках научного проекта № 1842-240012: „Перемагничивание магнитных наночастиц в сильных импульсных магнитных полях - новый подход к исследованию динамических эффектов, связанных с процессами намагничивания магнитных наночастиц“.

\section{Конфликт интересов}

Авторы заявляют, что у них нет конфликта интересов.

\section{Список литературы}

[1] J. Tućek, R. Zboril, A. Namai, S. Ohkoshi. Chem. Mater. 22, 6483 (2010).

[2] L. Machala, J. Tućek, R. Zboril. Chem. Mater. 23, 3255 (2011).

[3] M. Gich, A. Roig, C. Frontera, E. Molins, J. Sort, M. Popovici, G. Chouteau, D. Martın y Marero, J. Nogues. J. Appl. Phys. 98, 4, 044307 (2005).

[4] S. Sakurai, S. Kuroki, H. Tokoro, K. Hashimoto, S. Ohkoshi. Adv. Funct. Mater. 17, 2278 (2007).

[5] S. Ohkoshi, A. Namai, K. Imoto, M. Yoshikiyo, W. Tarora, K. Nakagawa, M. Komine, Y. Miyamoto, T. Nasu, S. Oka, H. Tokoro. Sci. Rep. 5, 14414, (2015).

[6] S. Ohkoshi, S. Kuroki, S. Sakurai, K. Matsumoto, K. Sato, S. Sasaki. Angew. Chem. Int. Ed. 46, 8392 (2007).

[7] А.И. Дмитриев, О.В. Коплак, А. Namai, H. Tokoro, S. Ohkoshi, Р.Б. Моргунов. ФТТ 56, 1735 (2014).

[8] S. Ohkoshi, A. Namai, T. Yamaoka, M. Yoshikiyo, K. Imoto, T. Nasu, S. Anan, Y. Umeta, K. Nakagawa, H. Tokoro. Sci. Rep. 6, 27212, (2016).

[9] A. Namai, S. Sakurai, M. Nakajima, T. Suemoto, K. Matsumoto, M. Goto, S. Sasaki, S. Ohkoshi. J. Am. Chem. Soc. 131, 1170 (2009).

[10] S. Ohkoshi, S. Kuroki, S. Sakurai, K. Matsumoto, K. Sato, S. Sasaki. Angew. Chem. Int. Ed. 46, 8392 (2007).

[11] J.L. García-Muñoz, A. Romaguera, F. Fauth, J. Nogués, M. Gich. Chem. Mater. 29, 9705 (2017).

[12] Д.А. Балаев, А.А. Дубровский, С.С. Якушкин, Г.А. Бухтиярова, О.Н. Мартьянов. ФТТ 61, 478 (2019).

[13] M. Kurmoo, J.-L. Rehspringer, A. Hutlova, C. D’Orlans, S. Vilminot, C. Estournes, D. Niznansky. Chem. Mater. 17, 1106 (2005).

[14] M. Gich, C. Frontera, A. Roig, E. Taboada, E. Molins, H.R. Rechenberg, J.D. Ardisson, W.A.A. Macedo, C. Ritter, V. Hardy, J. Sort, V. Skumryev, J. Nogués. Chem. Mater. 18, 3889 (2006).

[15] E. Tronc, C. Chaneac, J.P. Jolivet. J. Solid State Chem. 139, 1, 93 (1998).

[16] N.A. Usov, Yu.B. Grebenshchikov. J. Appl. Phys. 106, 023917 (2009).

[17] E.L. Verde, G.T. Landi, J.A. Gomes, M.H. Sousa, A.F. Bakuzis. J. Appl. Phys. 111, 123902 (2012).

[18] J. Carrey, B. Mehdaoui, M. Respaud. J. Appl. Phys. 109, 083921 (2011).

[19] А.С. Камзин, D.S. Nikam, S.H. Pawar. ФТT 59, 149 (2017).

[20] А.С. Камзин. ФТТ 58, 519 (2016).

[21] А.М. Шутый, Д.И. Семенцов. ФТТ 61, 1783 (2019).

[22] А.М. Шутый, Д.И. Семенцов. ЖЭТФ 156, 299 (2019).
[23] A.A. Dubrovskiy, D.A. Balaev, K.A. Shaykhutdinov, O.A. Bayukov, O.N. Pletnev, S.S. Yakushkin, G.A. Bukhtiyarova, O.N. Martyanov. J. Appl. Phys. 118 (2015).

[24] D.A. Balaev, I.S. Poperechny, A.A. Krasikov, K.A. Shaikhutdinov, A.A. Dubrovskiy, S.I. Popkov, A.D. Balaev, S.S. Yakushkin, G.A. Bukhtiyarova, O.N. Martyanov, Yu.L. Raikher. J. Appl. Phys. 117, 063908 (2015).

[25] I.S. Poperechny, Yu.L. Raikher, V.I. Stepanov. Phys. Rev. B 82, 174423 (2010).

[26] I.S. Poperechny, Yu.L. Raikher, V.I. Stepanov. Physica B 435, 58 (2014).

[27] S.S. Yakushkin, D.A. Balaev, A.A. Dubrovskiy, S.V. Semenov, Yu.V. Knyazev, O.A. Bayukov, V.L. Kirillov, R.D. Ivantsov, I.S. Edelman, O.N. Martyanov. Ceram. Int. 44, 17852 (2018).

[28] Ю.В. Князев, Д.А. Балаев, В.Л. Кириллов, О.А. Баюков, О.Н. Мартьянов. Письма ЖЭТФ 108, 558 (2018).

[29] А.Д. Балаев, Ю.В. Бояршинов, М.М. Карпенко, Б.П. Хрусталев. ПТЭ 3, 167 (1985).

[30] S.S. Yakushkin, A.A. Dubrovskiy, D.A. Balaev, K.A. Shaykhutdinov, G.A. Bukhtiyarova, O.N. Martyanov. J. Appl. Phys. 111, 44312 (2012).

[31] I. Edelman, J. Kliava, O. Ivanova, R. Ivantsov, D. Velikanov, V. Zaikovskii, E. Petrakovskaja, Y. Zubavichus, S. Stepanov. J. Non-Cryst. Solids 506, 68 (2019).

[32] O.S. Ivanova, R.D. Ivantsov, I.S. Edelman, E.A. Petrakovskaja, D.A. Velikanov, Y.V. Zubavichus, V.I. Zaikovskii, S.A. Stepanov. J. Magn. Magn. Mater. 401, 880 (2016).

[33] Д.А. Балаев, С.С. Якушкин, А.А. Дубровский, Г.А. Бухтиярова, К.А. Шайхутдинов, О.Н. Мартьянов. Письма ЖТФ 42, 23 (2016).

[34] D.A. Balaev, A.A. Krasikov, A.A. Dubrovskii, A.D. Balaev, S.I. Popkov, V.L. Kirillov, O.N. Martyanov. J. Supercond. Nov. Magn. 32, 405 (2019).

[35] Д.А. Балаев, А.А. Красиков, Д.А. Великанов, С.И. Попков, Н.В. Дубынин, С.В. Столяр, В.П. Ладыгина, Р.Н. Ярославцев. ФТТ 60, 1939 (2018).

[36] S. Mørup, D.E. Madsen, C. Fradsen, C.R.H. Bahl, M.F. Hansen. J. Phys.: Condens. Matter 19, 213202 (2007).

[37] Yu.L. Raikher, V.I. Stepanov. J. Phys.: Condens. Matter. 20, 204120 (2008).

[38] D.A. Balaev, A.A. Krasikov, A.A. Dubrovskiy, S.I. Popkov, S.V. Stolyar, O.A. Bayukov, R.S. Iskhakov, V.P. Ladygina, R.N. Yaroslavtsev. J. Magn. Magn. Mater. 410, 71 (2016).

[39] S.I. Popkov, A.A. Krasikov, D.A. Velikanov, V.L. Kirillov, O.N. Martyanov, D.A. Balaev. J. Magn. Magn. Mater. 483, 21 (2019).

[40] S.I. Popkov, A.A. Krasikov, A.A. Dubrovskiy, M.N. Volochaev, V.L. Kirillov, O.N. Martyanov, D.A. Balaev. J. Appl. Phys. 126, 103904 (2019).

[41] R.H. Kodama, A.E. Berkowitz. Phys. Rev. B 59, 6321 (1999).

[42] N.J.O. Silva, A. Millan, F. Palacio, E. Kampert, U. Zeitler, V.S. Amaral. Phys. Rev. B 79, 104405 (2009).

[43] D.A. Balaev, A.A. Krasikov, A.A. Dubrovskiy, S.I. Popkov, S.V. Stolyar, R.S. Iskhakov, V.P. Ladygina, R.N. Yaroslavtsev. J. Appl. Phys. 120, 183903 (2016).

[44] E.C. Stoner, E.P. Wohlfarth. Philos. Trans. Roy. Soc. London. A 240, 599 (1948).

[45] P. Brázda, D. Nižănsky, J.-L. Rehspringer, J.P. Vejpravová. J. Sol-Gel Sci. Technol. 51, 78 (2009).

Редактор К.В. Емцев 